\title{
SPL Unit of Presentation Terminology
}

National Cancer Institute

\section{Source}

National Cancer Institute. SPL Unit of Presentation Terminology. NCI Thesaurus. Code C87300.

Terminology that encompasses dosage form, package type, and unit of measurement (aka potency) terms used in supporting regulatory submissions in electronic format drug establishment registration and drug listing. 